available resources. As such the team are investigating using an electronic sleep diary, integrating with actigraphy and simplifying the whole process. The first stage of this process was to ask the stakeholders their opinions of sleep diaries and actigraphy.

Methods Stakeholders were asked about sleep diaries and actigraphy considering all aspects of the process from when to request a referral and organising collection, completing a diary and wearing actigraphy, through to analysing and interpreting results. Questions asked were appropriate to the different stakeholder groups involved. Families and local hospital staff were interviewed and British Paediatric Sleep Society (BPSA) members and families undergoing actigraphy were sent appropriate brief questionnaires relevant to their part of the pathway [Service evaluation SE1347].

Results 47 stakeholders provided feedback. Interviews were conducted with 14 staff, 9 parents and 3 young people. Additional questionnaires were completed by 8 families undergoing actigraphy and 13 BPSA responders. A summary of the most common responses is shown in table 1 . Most institutions were issuing sleep diaries and actigraphy to specific patient groups due to limited resources.

Discussion This stakeholder engagement provides useful information to improve the use of sleep diaries and actigraphy within our paediatric clinical setting. If the process can be simplified then more sleep diary and actigraphy usage can occur at the triage stage of the sleep referral process and prevent unnecessary sleep studies, benefitting both families and limited resources. Feedback received confirms the current solution for paediatric sleep diaries and actigraphy is not ideal and there is a need to identify new approaches to increase usability, acceptability and stakeholder's satisfaction.

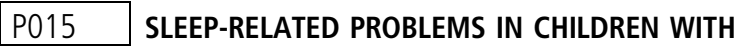 OSTEOGENESIS IMPERFECTA}

Claire Hill, Kieran Murphy, Ruth Kingshott*, Nicki Barker. Sheffield Children's NHS Foundation Trust, Sheffield Children's Hospital, Western Bank, Sheffield, UK

\subsection{6/bmjresp-2019-bssconf.15}

Introduction Osteogenesis Imperfecta (OI) is a genetic disorder affecting 1 in 10,000 births with a wide variability in phenotypes. Clinical manifestations include; recurrent fractures, bone pain, varying degrees of short stature and deformity, scoliosis, kyphosis, and respiratory failure in the severest types.
Sleep disorders are often under-acknowledged and are frequently more problematic in children with chronic illnesses. Children with OI are likely to complain of poor sleep quality due to pain, night sweats, structural changes in the chest and spine restricting the lungs, and soft tissues changes leading to decreased muscle tone, which may be linked to obstructive sleep apnoea.

The aim of the current project was to examine whether sleep-related problems are a significant issue to patients with OI at Sheffield Children's Hospital.

Methods A convenience sample of children with OI and their carers completed questionnaires designed by the project team to capture sleep and respiratory-related problems [service evaluation SE1090].

Results 55 patients (26 female; mean age 12.77 yrs; range 3.117.3) and carers completed the questionnaires during routine admission or clinic appointment. Participants were classified by OI type; 32-mild, 13-moderate, 10-severe.

$67 \%$ reported difficulty in getting off to sleep. $36 \%$ of participants reported waking in the night, with discomfort $(44 \%)$ and feeling too hot $(36 \%)$ being the most common causes. Frequency of night time waking ranged from $0-4$ times a night. $27 \%$ of participants reported snoring.

Discussion This service evaluation highlighted that OI patients are reporting sleep-related issues and further focus on sleep in the clinical history may be of importance. A pilot study examining the feasibility of polysomnography and sleep-related questionnaires in children with OI is currently underway to further research sleep in this cohort.

\section{P016 USING NON-INVASIVE THERMAL IMAGING FOR APNOEA DETECTION}

${ }^{1}$ Usman Muhammad, ${ }^{1}$ Ruth Evans, ${ }^{1}$ Reza Saatchi, ${ }^{2}$ Ruth Kingshott* ${ }^{*}{ }^{2}$ Heather Elphick. ${ }^{1}$ Materials and Engineering Research Institute, Sheffield Hallam University, Sheffield, UK; ${ }^{2}$ Sheffield Children's NHS Foundation Trust, Sheffield, UK

\subsection{6/bmjresp-2019-bssconf.16}

Introduction The current gold standard sensors to measure airflow in apnoea and hypopnoea detection are the oronasal thermal airflow sensor and the nasal pressure transducer, respectively. ${ }^{1}$ Due to the contact nature of these sensors in the nasal region, they are poorly tolerated by children. In a recent audit, we found that $50 \%$ of children refused the nasal pressure sensor due to its invasiveness, and of those that

\begin{tabular}{|c|c|c|c|c|c|c|}
\hline \multirow{2}{*}{$\boldsymbol{N}$} & \multicolumn{3}{|c|}{ Males } & \multicolumn{3}{c|}{ Females } \\
\cline { 2 - 7 } & $\begin{array}{c}\text { Elementary } \\
\text { (Parent- } \\
\text { report) }\end{array}$ & $\begin{array}{c}\text { Intermediate } \\
\text { (Self-report) }\end{array}$ & $\begin{array}{c}\text { Secondary } \\
\text { (Self- } \\
\text { report) }\end{array}$ & $\begin{array}{c}\text { Elementary } \\
\text { (Parent- } \\
\text { report) }\end{array}$ & $\begin{array}{c}\text { Intermediate } \\
\text { (Self-report) }\end{array}$ & $\begin{array}{c}\text { Secondary } \\
\text { (Self- } \\
\text { report) }\end{array}$ \\
\cline { 2 - 7 } & 50 & 39 & 50 & 50 & 50 & 50 \\
\hline Mean age & 10 & 14 & 16 & 10 & 14 & 16 \\
\hline Mean score CSHQ & 48 & 53 & 48 & 49 & 55 & 54 \\
\hline $\begin{array}{c}\text { Percentage of sleep } \\
\text { difficulties }\end{array}$ & $86 \%$ & $95 \%$ & $86 \%$ & $86 \%$ & $100 \%$ & $100 \%$ \\
Score of 41 or above & & & & & & \\
\hline Average sleep time & $9 \mathrm{pm}$ & $11 \mathrm{pm}$ & $11 \mathrm{pm}$ & $10 \mathrm{pm}$ & $11 \mathrm{pm}$ & $11 \mathrm{pm}$ \\
\hline Average wakeup time & $6 \mathrm{am}$ & $6 \mathrm{am}$ & $6 \mathrm{am}$ & $5 \mathrm{am}$ & $5 \mathrm{am}$ & $5 \mathrm{am}$ \\
\hline Average sleep duration & 9 & 7 & 7 & 7 & 6 & 6 \\
\hline
\end{tabular}

Abstract P016 Figure 1 Sample characteristics and the CSHQ scores 
accepted it, 64\% removed the prongs over the course of the night.

The aim of the current study was to evaluate a non-contact method to monitor respiration by developing infrared thermal imaging, whereby temperature fluctuations associated with respiration are measured and correlated with airflow.

Methods 11 healthy adult volunteers participated following University Ethics. Respiratory signals were recorded over four simulated apnoea scenarios resembling normal respiration; central; obstructive and hypopnoeic pauses. Simulated apnoeas were measured via nasal pressure cannula and respiratory inductance bands [SOMNOtouchTM RESP; SOMNOmedics, Germany] versus thermal imaging techniques.

Results $70 \%$ of the apnoea episodes correlated with airflow sensor readings (Example trace in figure 1). In $16 \%$ of recordings the subject's head position did not allow correct identification of the region of interest (i.e. the nostrils). For the remaining $14 \%$ of cases there was partial agreement between the thermal measurements and nasal pressure readings.

Discussion These results indicate thermal imaging may be valuable as a detection tool for sleep apnoea, particularly in the case of paediatric patients whose tolerance for contact nasal sensors is poor.

\section{REFERENCE}

1. Berry RB, Albertario $C L$, Harding SM, et al. for the American Academy of Sleep Medicine. The AASM Manual for the Scoring of Sleep and Associated Events: Rules, Terminology and Technical Specifications. Version 2.5. Darien, IL: American Academy of Sleep Medicine, 2018.

\section{P017 DIFFERENCES IN GENETIC RISK FOR INSOMNIA, HYPERSOMNIA AND CHRONOTYPE IN BIPOLAR DISORDER SUBTYPES}

\begin{abstract}
1,2Katie JS Lewis*, ${ }^{1}$ Alexander Richards, ${ }^{3}$ Robert Karlsson, ${ }^{1}$ Ganna Leonenko, ${ }^{4}$ Samuel E Jones, ${ }^{5,6,7}$ Hannah Jones, ${ }^{8}$ Katherine Gordon-Smith, ${ }^{1}$ Liz Forty, ${ }^{1}$ Valentina Escott-Price, ${ }^{1,2}{ }^{3}$ Michael J Owen, ${ }^{4}$ Michael N Weedon, ${ }^{8}$ Lisa Jones, ${ }^{1,2}$ Nick Craddock, ${ }^{1,2}$ lan Jones, ${ }^{3,9}$ Mikael Landén, ${ }^{1,2}$ Michael C O'Donovan, ${ }^{1,2}$ Arianna Di Florio. ${ }^{1}$ Medical Research Council Centre for Neuropsychiatric Genetics and Genomics, Institute of Psychological Medicine and Clinical Neurosciences, School of Medicine, Cardiff University, Cardiff, UK; ${ }^{2}$ National Centre for Mental Health, Cardiff University, Cardiff, UK; ${ }^{3}$ Department of Medical Epidemiology and Biostatistics, Karolinska Institutet, Stockholm, Sweden; ${ }^{4}$ Genetics of Complex Traits, University of Exeter Medical School, Exeter, UK; ${ }^{5}$ Centre for Academic Mental Health, Population Health Sciences, Bristol Medical School, University of Bristol, Bristol, UK; ${ }^{6}$ Medical Research Council (MRC) Integrative Epidemiology Unit (IEU), University of Bristol, Bristol, UK; ${ }^{7}$ NIHR Biomedical Research Centre at University Hospitals Bristol NHS Foundation Trust and the University of Bristol, Bristol, UK; ${ }^{8}$ Department of Psychological Medicine, University of Worcester, Worcester, UK; ${ }^{9}$ Institute of Neuroscience and Physiology, Sahlgenska Academy at the Gothenburg University, Gothenburg, Gothenburg, Sweden
\end{abstract}

10.1136/bmiresp-2019-bssconf.17

Introduction Insomnia, hypersomnia and evening chronotype are common in bipolar disorder (BD) but research examining the role of genetics is mixed. Stratifying by bipolar subtypes could elucidate this relationship and inform sleep and BD research. Aim: to determine whether genetic liability to insomnia, hypersomnia and chronotype differ between bipolar subtypes (type $1, \mathrm{BD}-\mathrm{I}$ or type $2, \mathrm{BD}-\mathrm{II}$ ).

Method Case-control study of 4672 participants with BD (67\% female, 3404 BD-I, 1268 BD-II) enrolled in the Bipolar Disorder Research Network and 5714 controls (49\% female) recruited from the 1958 British Birth Cohort and UK Blood
Service. All participants were of European ancestry. BD subtypes were determined by semi-structured psychiatric interview and case notes. Genetic liability to sleep traits was assessed using genetic risk scores (GRS), which were derived using alleles from genome-wide association studies of insomnia, sleep duration, daytime sleepiness and chronotype. Analyses used multinomial regression to determine whether GRS for insomnia, hypersomnia (daytime sleepiness or sleep duration) and chronotype were associated with BD-I or BD-II when compared to controls.

Results Insomnia GRS were associated with increased risk of BD-II $(\mathrm{RR}=1.14,95 \% \mathrm{CI}=1.07-1.21, \mathrm{P}=8.26 \times 10-5)$ but not $\mathrm{BD}-\mathrm{I}(\mathrm{RR}=0.98,95 \% \mathrm{CI}=0.94-1.03, \mathrm{P}=0.409)$ relative to controls. Sleep duration GRS were associated with increased relative risk of $\mathrm{BD}-\mathrm{I}(\mathrm{RR}=1.10,95 \% \mathrm{CI}=1.06-1.15$, $\mathrm{P}=1.13 \times 10-5)$, but not $\mathrm{BD}-\mathrm{II}(\mathrm{RR}=0.99,95 \% \mathrm{CI}=0.93-$ $1.06, \mathrm{P}=0.818$ ). Daytime sleepiness and chronotype GRS did not distinguish bipolar subtypes.

Discussion Bipolar subtypes differ in genetic liability to insomnia and sleep duration, providing further evidence that bipolar subtypes should be considered separately in research on sleep in BD. The distinct findings for sleep duration and daytime sleepiness support existing literature suggesting that these are distinct subtypes of hypersomnia.

\section{P018 A COMPARISON OF SLEEP PARAMETERS MEASURED BY LIMITED MULTICHANNEL POLYSOMNOGRAPHY AND FULL POLYSOMNOGRAPHY}

Demi Callis*, Liam O'Reilly, Michelle Goodlad. NHS, Coventry, UK

\subsection{6/bmjresp-2019-bssconf. 18}

Introduction Inpatient full polysomnography (PSG) is the gold standard diagnostic to identify obstructive sleep apnoea syndrome (OSAS)1. Due to healthcare resources and utilisation many sleep centres employ limited multichannel sleep testing (MC) at the patient's home. Both diagnostic tests provide measurements of oxygen desaturation index (ODI), apnoeahypopnoea index (AHI) and oxygen saturation (Sp02). The sleep studies however are subject to factors that may influence sleep quality, including environmental effects (location of performance and staff versus patient setup) that may influence overall sleep study scoring.

Methods All patients referred to the CSU for investigation of OSAS who subsequently performed a PSG were included in this observational study. Patients who were symptomatic (Epworth score $\geq 11$ ) and received a diagnosis of mild OSAS (AHI 5-14/hr) continued to PSG. MC were performed using NOX T3 and PSG using NOX A1 (Nox Medical, Katrínartún, Iceland). Sleep scoring was in accordance with AASM guidelines version 2.3. Comparisons of data sets was performed using SPSS statistical software.

Results Patient demographics are shown in table 1 . In total 8 patients were included. All patients scored a higher AHI on PSG compared to MC. Mean PSG AHI and ODI were significantly higher than MC $(18.48 / \mathrm{hr} ; 8.1 / \mathrm{hr}$ and $17.68 /$ hr; 7.08/hr, p $\leq 0.001$ respectively). Sp02 was comparable between PSG and MC (92\% and 94\%, p=0.0135, respectively). [Figure 1].

Discussion Data from this single centre, small sample study shows higher AHI and ODI from PSG compared to MC in 